\title{
Surgical interventions for primary central nervous system lymphoma
}

\author{
James B. Elder, M.D., And Thomas C. Chen, M.D., Ph.D. \\ Department of Neurosurgery, University of Southern California, Los Angeles, California
}

\begin{abstract}
$\checkmark$ Early diagnosis is central to proper management of primary central nervous system lymphomas (PCNSLs). Surgical intervention hinges on initial entertainment of a diagnosis of a PCNSL, based on acute neurological presentation and neuroimaging findings. Unless there is an urgent need for surgical decompression, a biopsy to obtain a diagnosis of PCNSL is the first step in surgical management. Repeated biopsy may be necessary in patients who have received preoperative steroid therapy. Patients with PCNSL may also present with leptomeningeal involvement, resulting in the need for an Ommaya reservoir for intrathecal chemotherapy. In cases in which hydrocephalus develops, placement of a ventriculoperitoneal shunt may be necessary. Two case studies are presented to highlight the role of surgical intervention in PCNSL.
\end{abstract}

KEY WORDS • primary central nervous system lymphoma • surgery $・$ review

$\mathrm{P}$ RIMARY central nervous system lymphomas historically have accounted for approximately $2 \%$ of primary brain tumors. ${ }^{2,10}$ The tumor has a higher incidence among immunocompromised patients, and it is the most common intracranial neoplasm in patients with AIDS. ${ }^{2,6}$ The body of literature regarding PCNSL strongly supports a protocol for stereotactic biopsy to establish diagnosis, followed by chemotherapy and radiation therapy tailored to treat the histological subtype of the tumor. ${ }^{3}$ Although rates of response to chemotherapy and radiotherapy approach $80 \%$, the treatments are rarely curative, and patients with PCNSL generally survive between 1 and 4 years after diagnosis. ${ }^{13}$ Craniotomy for debulking or resection of the tumor is widely considered to have no role in the treatment of PCNSL. ${ }^{4}$ In this review, we discuss considerations regarding the surgical management of PCNSL, including proper patient selection, preoperative workup, and the role and methods of stereotactic biopsy, as well as summarizing the literature on open craniotomy. Case studies from the authors' experience with PCNSL are also presented.

\section{Diagnosis and Treatment}

\section{Clinical Presentation}

The clinical presentation of patients with PCNSL varies depending on the location and size of the tumor, as well as the immunological status of the patient. ${ }^{14}$ Without treatment, PCNSL is rapidly fatal. ${ }^{19}$ In immunocompetent pa-

Abbreviations used in this paper: CNS = central nervous system; $\mathrm{CSF}=$ cerebrospinal fluid; $\mathrm{CT}=$ computed tomography; FDG = $\left[{ }^{18} \mathrm{~F}\right]$ fluorodeoxyglucose; $\mathrm{MR}=$ magnetic resonance; $\mathrm{PCNSL}=$ primary CNS lymphoma; PET = positron emission tomography. tients with PCNSL, the median age at presentation is 55 years, and the ratio of male to female patients has been reported as ranging from 1.2:1 to 1.7:1. In immunosuppressed patients, the median age at presentation is 40 years. ${ }^{13,14}$

Patients with PCNSL typically present with neurological signs and symptoms-focal neurological deficit, seizures, clinical signs of intracranial hypertension, neuropsychiatric symptoms, and/or visual complaints-that can develop rapidly (over 2 to 3 weeks) or more slowly (over a period of several months). Neurological signs may be preceded by systemic symptoms such as gastrointestinal or febrile respiratory illness in up to $15 \%$ of patients. ${ }^{3,16}$ The rapid development of neurological symptoms may be a key indicator that a patient with a mass lesion is suffering from lymphoma.

\section{Preoperative Management}

Baseline Evaluation. Guidelines for standard baseline evaluation of cases of newly diagnosed PCNSL have been published by the International PCNSL Collaborative Group. ${ }^{1}$ Determination of the full extent of the disease is important for guiding therapy. Primary central nervous system lymphoma is a rare form of extranodal non-Hodgkin lymphoma that may involve the brain or any part of the craniospinal axis, including the eyes, spinal cord, leptomeninges, and CSF. Therefore, all areas must be evaluated for the presence of tumor prior to therapeutic intervention. Continued evaluation of patients presenting with PCNSL may reveal extraneural tumor. ${ }^{9}$ Systemic disease should also be ruled out prior to cranial biopsy, because the finding of extracranial disease can obviate the need for a cranial procedure. Current recommendations include CT scans of the chest, abdomen, and pelvis, as well as a bone marrow biopsy with aspirate. Whole-body PET may also be incorporated into the systemic workup. 
Timing of Steroid Therapy. Clinical improvement in patients with PCNSL after initiation of glucocorticoid therapy is largely due to decreased edema in the surrounding brain tissue. Steroid agents also play an important role in the medical therapy for PCNSL because of their lympholytic effects. Glucocorticoids may even cause complete resolution of lesions within a few days after initiation of treatment. ${ }^{11}$

In contrast to patients with other CNS lesions, however, patients with PCNSL should not receive corticosteroid agents prior to tissue diagnosis because the lympholytic effect of these medications may result in a nondiagnostic biopsy. ${ }^{26,11}$ If a stereotactic brain biopsy is performed while a patient is receiving steroid therapy, the histopathological findings may be interpreted as inflammatory changes due to the rapid killing of B cells. Steroid treatment may also result in the findings of demyelination and the presence of $\mathrm{T}$ cells within the specimen, which can lead to a false negative biopsy result.

Developing an early clinical and neuroimaging suspicion of PCNSL in cases of newly diagnosed brain tumors is crucial to maximizing the success rate of the stereotactic biopsy. If a tissue diagnosis has not yet been obtained in a given case, but the steroid therapy has already been initiated, the biopsy procedure should be delayed. There is currently no specific recommendation for the length of this delay. One case report describes dramatic decrease in the size of a tumor after 12 days of glucocorticoid therapy. A stereotactic biopsy was negative for tumor, but withholding steroid treatment for 5 days resulted in distinct tumor regrowth as demonstrated on MR images, and the subsequent biopsy was positive for PCNSL. ${ }^{1}$ In our practice, we try to avoid administering steroid agents for as long as 2 weeks prior to a repeated biopsy, provided the patient's condition remains stable. In certain cases, we use imaging to predict when a repeated biopsy can be performed. If a patient who was previously treated with steroids experiences an increase in tumor size after cessation of steroid therapy, or if a tumor that previously appeared "cold" on a PET scan appears "hot," we would consider that sufficient time had elapsed for the repeated biopsy to be performed.

\section{Imaging Modalities}

Computed Tomography. Because patients often present with acute or subacute neurological symptoms, the initial diagnostic image is most often a noncontrast CT scan of the brain. This scan typically reveals a lesion that is isodense to hyperdense compared with gray matter and is usually surrounded by significant edema. Hemorrhage associated with the tumor may be seen within or adjacent to the lesion, or as blood in the ventricular system.

Intravenous administration of contrast material reveals significant enhancement of the lesion. Other CT findings may include mass effect, with compression of adjacent structures such as the lateral ventricle, and midline shift. Compression of ventricles may lead to ventriculomegaly, generalized hydrocephalus, or loculation of a portion of the ventricular system due to outflow obstruction. For these reasons, transependymal edema may also be present.

Magnetic Resonance Imaging. Brain MR imaging - with and without intravenous administration of gadolinium- remains the modality of choice for finer anatomical discrimination of the extent of lesions and planning therapeutic interventions such as biopsy or surgical decompression. Noncontrast imaging reveals a mass isointense to hypointense on $\mathrm{T}_{1}$-weighted and $\mathrm{T}_{2}$-weighted sequences. As in CT images, findings such as edema, hydrocephalus, mass effect on surrounding structures, and midline shift may be observed. However, MR imaging typically offers much improved anatomical detail for assessing the extent of edema and the presence of transependymal flow of CSF.

Intravenous administration of gadolinium reveals bright contrast enhancement of the tumor. The tumor borders are most commonly well demarcated and brightly enhancing. Necrosis may be present within the tumor and will appear hypointense compared to the outer layer of living tumor cells. Küker and colleagues ${ }^{18}$ evaluated MR images of 100 patients with newly diagnosed PCNSL. They found that lesions were most often solitary $(65 \%)$ and enhanced strongly on administration of contrast medium (85\%). The most common location was hemispheric (38\%), followed by thalamus/basal ganglia (16\%), corpus callosum (14\%), periventricular $(12 \%)$, and cerebellar $(9 \%)$. All but one patient had at least one PCNSL lesion in contact with the CSF space. Enhancement along Virchow-Robin spaces is considered a highly specific feature of PCNSL. Overall, only $1 \%$ of tumors lacked contrast enhancement.

For establishing an initial imaging suspicion of PCNSL, determining the anatomical extent of the tumor, and planning surgical intervention, MR imaging with gadolinium enhancement is the study of choice. Patients who have a contraindication to MR imaging should undergo contrastenhanced CT.

Magnetic Resonance Spectroscopy. Little data exist regarding findings of MR spectroscopy in patients with primary CNS lymphoma. One report ${ }^{18}$ presents preliminary results for four patients with known PCNSL who underwent MR spectroscopy as part of their initial workup. The studies showed a near complete loss of the $N$-acetylaspartate peak, a decreased creatine peak, and increases in the choline, lactate, and lipids/macromolecules peaks. Although the findings were considered similar for all four patients, further studies are needed to elucidate the usefulness of this technique either for narrowing the preoperative differential diagnosis or for monitoring the effects of therapy.

Positron Emission Tomography. Positron emission tomography of the brain can provide information about blood-brain barrier permeability and drug distribution as well as brain metabolism and perfusion by evaluating uptake of radiolabeled tracer substances. Tracer activity in the target region ( $\mathrm{T}$ ) is compared to normal brain parenchyma in the contralateral hemisphere $(\mathrm{C})$. One example of a tracer is ${ }^{18} \mathrm{FDG}$, which correlates to glycolysis as an indicator of metabolic activity. Increased ${ }^{18} \mathrm{FDG}$ uptake $(\mathrm{T} / \mathrm{C}>1)$ is seen in all malignant brain tumors, but the ratio may be highest in PCNSLs, which have a ratio comparable to (probably a little higher than) that found in metastases and considerably higher than that found in gliomas. ${ }^{21}$

In patients with HIV who present with ring-enhancing lesions, PET can also be used to distinguish between toxoplasmosis and PCNSL. In contrast to PCNSL, toxoplasmosis lesions are relatively hypometabolic and therefore 
show lower ${ }^{18} \mathrm{FDG}$ uptake. Although PET has been shown to be a useful tool for staging other types of cancer, its role in determining the extent of disease in patients with PCNSL is not yet clear. ${ }^{4}$

\section{Differential Diagnosis}

In cases in which the clinical and imaging presentation is consistent with PCNSL, a broad range of diagnoses must be considered. Neoplasms such as glioblastoma multiforme and metastases may mimic PCNSL both clinically and in terms of imaging characteristics, and they affect immunocompetent patients of similar ages. Thus, a biopsy and tissue diagnosis are crucial. Inflammatory diseases such as multiple sclerosis or sarcoidosis and neurodegenerative diseases may also present with similar clinical findings in the immunocompetent patient. Because the lesions in PCNSL often are located in the periventricular region, long-term personality changes may, in the absence of appropriate imaging studies, lead to a diagnosis of a neurodegenerative disorder. ${ }^{14}$

In patients with AIDS, it is also important to consider toxoplasmosis in the differential diagnosis. However, when symptoms are caused by toxoplasmosis, appropriate therapy should lead to rapid clinical improvement; PCNSL must be considered if clinical deterioration persists despite antitoxoplasmosis therapy. ${ }^{8}$

\section{Surgical Considerations}

Stereotactic Biopsy. Biopsies of intracranial PCNSLs can be performed via frame-based or frameless stereotactic protocols. The choice depends primarily on the surgeon's experience with the procedures and the availability of the necessary equipment. In general, we prefer to perform frame-based biopsies in cases involving small $(<2 \mathrm{~cm})$, deep midline lesions and in tumors that enhance well with contrast. Frameless systems are often used for a tumor in the temporal lobe (where it is more difficult to achieve a good trajectory with a frame-based system) and for larger lesions $(>2 \mathrm{~cm})$.

When we perform a frame-based stereotactic biopsy, we initially fix a CT-compatible stereotactic base ring to the patient's head while he or she is in a state of conscious sedation and after the application of a local anesthetic. A localizing frame is placed on top of the base ring. The patient is then taken from the operating room for a contrastenhanced CT scan of the brain. Coordinates (x, y) for the biopsy target as well as pixel coordinates of the nine localizer rods are chosen and entered into a Epson HX-20 computer. The program calculates the anteroposterior, lateral, and vertical coordinates for the biopsy. After the patient is returned to the operating room, a general anesthetic is administered via a mask or endotracheal tube. The base ring is secured to the operating table using a Mayfield holder, and the localizing frame is removed. The target coordinates generated by the computer are applied to the phantom base and confirmed on the arc frame, which is then attached to the base ring. These coordinates are used to guide the biopsy trajectory and depth. A $1-\mathrm{cm}$ incision and bur hole are made based on this trajectory, and the biopsy is performed using small biopsy forceps, following the same trajectory. The biopsy samples are sent for pathological analysis. Frozen section of the lesion with standard hematoxylin and eosin staining is usually diagnostic in cases in which PCNSL is strongly suspected. Histological and immunohistochemical analysis of paraffin-embedded tissue is performed using the following stains and antibodies: hematoxylin and eosin, glial fibrillary acidic protein (a marker for glial neoplasms), CD3 (a T-cell marker), CD20 (a B-cell marker), CD45 (a common leukocyte antigen, which stains both T and B cells), and keratin (a marker for carcinoma). These studies solidify the diagnosis of PCNSL and may further categorize the tumor based on immunological cell surface markers. This analysis may be important when designing future therapeutic treatments specific to tumor subsets. After positive biopsy is achieved, the frame is removed and the incision is irrigated and closed.

Frameless stereotactic biopsy is performed using many principles of the frame-based approach, with the following differences. Before the frameless procedure, a series of fiducials that are compatible with either CT or MR imaging are placed on the patient's scalp. The patient then undergoes a volumetric CT or MR imaging study of the brain with contrast enhancement, and the resulting images are loaded into a neuronavigation station (for example, Stealth or BrainLab). The patient's head is secured to the operating table using a Mayfield clamp, and the fiducial points are registered with the corresponding points on the neuronavigation system. Thus, the three-dimensional image on the navigation system, which includes the intracranial contents and, more importantly, the tumor, is coordinated with the patient. An alternative to the fiducial method requires only a preoperative volumetric MR imaging study with contrast. No fiducials are used. Instead, a separate wand uses facial landmarks to register the patient's anatomy with the MR image on the navigation system.

Use of a navigational device and registration points adjacent to the operative field allows planning of the biopsy trajectory and depth. Again, a small incision and bur hole are made based on this preoperative planning. A small biopsy sample is obtained using a combination of cranial attachments and probes, configured according to the operative plan. Once a positive biopsy has been achieved, the system is removed and the incision is irrigated and closed. An immediate noncontrast CT study of the head is obtained to rule out intracranial bleeding due to the procedure. As can be surmised from the description, stereotactic biopsy represents a minimally invasive means of obtaining samples for tissue diagnosis.

Because the procedure is brief, the majority of patients return quickly to their preoperative neurological baseline. After surgery, glucocorticoid therapy may be initiated. The resulting reduction in peritumoral edema typically results in improvement of neurological symptoms. Patients are given anticonvulsant medication at the time of surgery and for 1 week after the procedure. Patients who experience new-onset seizures due to their lymphomas are treated with anticonvulsant therapy for at least 3 months. Postoperative management depends on the severity of neurological symptoms and is governed largely by physical and speech therapy departments. Further oncological management of individual cases is dictated largely by the histopathological characteristics of the tumor, systemic findings from the preoperative workup, and the patient's HIV status. 
Catheter Reservoir Placement. An Ommaya reservoir can be implanted if intrathecal delivery of chemotherapy is desired (for instance, in patients with leptomeningeal metastasis). Intrathecal methotrexate or cytarabine (DepoCyt) can be delivered into the reservoir. Patients with hydrocephalus should not receive an Ommaya reservoir.

The procedure begins with a small incision in the frontal region, most commonly on the right side. A bur hole is placed in the midpupillary line approximately $1 \mathrm{~cm}$ anterior to the coronal suture. A small hole is created in the dura mater, and a ventricular catheter is inserted into the frontal horn of the lateral ventricle. This catheter is then secured to the Ommaya reservoir, which is itself secured to the periosteum of the skull. The wound is irrigated and closed. The advantage of an Ommaya reservoir over other catheter reservoirs is that its large size allows for easier access.

Ventriculoperitoneal Shunt Placement. Primary CNS lymphomas can cause generalized hydrocephalus or loculation of part of the ventricular system. Mass effect from the tumor or leptomeningeal involvement by tumor or inflammation may lead to obstruction of CSF outflow. In these cases, placement of a ventriculoperitoneal shunt is indicated for diversion of CSF.

A ventricular catheter is placed in a similar fashion as for the catheter reservoir. It is then connected to a valve that controls the pressure at which CSF drains from the intracranial cavity. The valve may be set at a fixed pressure (for example, $10 \mathrm{~mm} \mathrm{Hg}$ ) or it may be programmable, in which case a range of pressure settings can be chosen during surgery or at any time thereafter. The valve is connected to the peritoneal tubing, which is tunneled under the skin behind the ear, in the lateral part of the neck, over the chest wall, and into the peritoneal cavity. A stab incision behind the ear is required to tunnel the peritoneal tubing. A third incision in the upper abdominal region is required for placement of the peritoneal tubing in the peritoneal cavity. A common alternative, especially in pediatric cases, is placement of the ventricular catheter in a parietooccipital trajectory. In these cases, a stab incision behind the ear is often not required.

There have been cases in which a patient initially had an Ommaya reservoir placed for treatment of leptomeningeal disease and hydrocephalus subsequently developed. In such cases, we have placed a separate ventriculoperitoneal shunt with a programmable valve. At the time of the intrathecal injection, the valve pressure is raised to its highest level (to decrease runoff into the peritoneal cavity). The valve pressure is then lowered to its normal level the following day.

Craniotomy. Surgical resection or debulking of PCNSL can be performed with or without frameless stereotactic localization, which we have described in a previous section of this article. The surgical incision depends on the location of the tumor. Effort is made to minimize the size of the incision and bone flap and to minimize risk of injury to eloquent areas of the brain. Microsurgical techniques allow the surgeon to minimize disruption of normal brain parenchyma during exposure of the tumor. Once exposed, the tumor is removed piecemeal, commonly with an ultrasonic aspirator. After careful hemostasis of the resection bed is achieved, the wound is closed.

\section{Illustrative Cases}

Two case reports illustrate many of the details discussed thus far in this article. Steroids were withheld in both cases until tissue diagnosis was achieved via stereotactic brain biopsies. Both patients were treated with chemotherapy and radiation therapy after diagnosis. The cases differed, however, in their postoperative courses. The patient in Case 1 required a craniotomy due to sudden neurological deterioration. He had no evidence of disease on follow-up neuroimaging for 9 months after surgery, but then developed multifocal recurrence. The patient in Case 2 underwent a stereotactic biopsy, followed by placement of an Ommaya reservoir, and responded well to chemotherapy. However, a surveillance MR imaging study 4 months after the biopsy showed recurrence.

\section{Case 1}

This 51-year-old man presented to his primary care physician with a 1-month history of progressive headache and weakness in his left hand. The outpatient workup included an MR imaging study of the brain, which was suspicious for lymphoma. The patient was scheduled for a stereotactic brain biopsy as an outpatient. However, he presented to the hospital prior to his scheduled surgery date due to a 1-week history of worsening left hemiparesis and mild confusion (forgetting where he was and what he was doing). A head CT scan and MR imaging (Fig. 1A) of the brain showed a large, enhancing mass in the right hemisphere. Given that lymphoma was suspected, steroid therapy was withheld. A stereotactic biopsy of the lesion was performed, and B-cell lymphoma was diagnosed.

After the biopsy, a workup for extent of disease was undertaken. The patient underwent CT scanning of the chest, abdomen, and pelvis and an ophthalmologic evaluation. No evidence of additional disease was discovered, and the results of an HIV test were likewise negative. Moderate left hemiparesis and intermittent confusion persisted after the biopsy. The patient was transferred to the nearby cancer hospital for further treatment.

One week after beginning chemotherapy, the patient was transferred back to our hospital due to deteriorating neurological status. He had become increasingly somnolent over the preceding night and was not following commands or verbalizing responses to questions. Diuresis resulted in only mild improvement in his symptoms. Emergency MR imaging (Fig. 1B) showed an increase in tumor size, edema, and right-to-left shift. Given the acute deterioration and worrisome neuroimaging findings, we decided to perform a craniotomy for tumor resection. Within 48 hours after surgery, the patient returned to his prebiopsy neurological baseline. Over the next 10 days, he experienced moderate improvement in his strength and was ultimately transferred to a rehabilitation facility.

Follow-up imaging was obtained at 1,6, and 9 months after surgery. Initial images showed no evidence of residual tumor. However, 9 months after craniotomy, MR imaging demonstrated multiple small enhancing lesions not contiguous with the original tumor resection cavity (Fig. $1 \mathrm{C}$ and D).

\section{Case 2}

This 61-year-old woman with a 15-year history of de- 

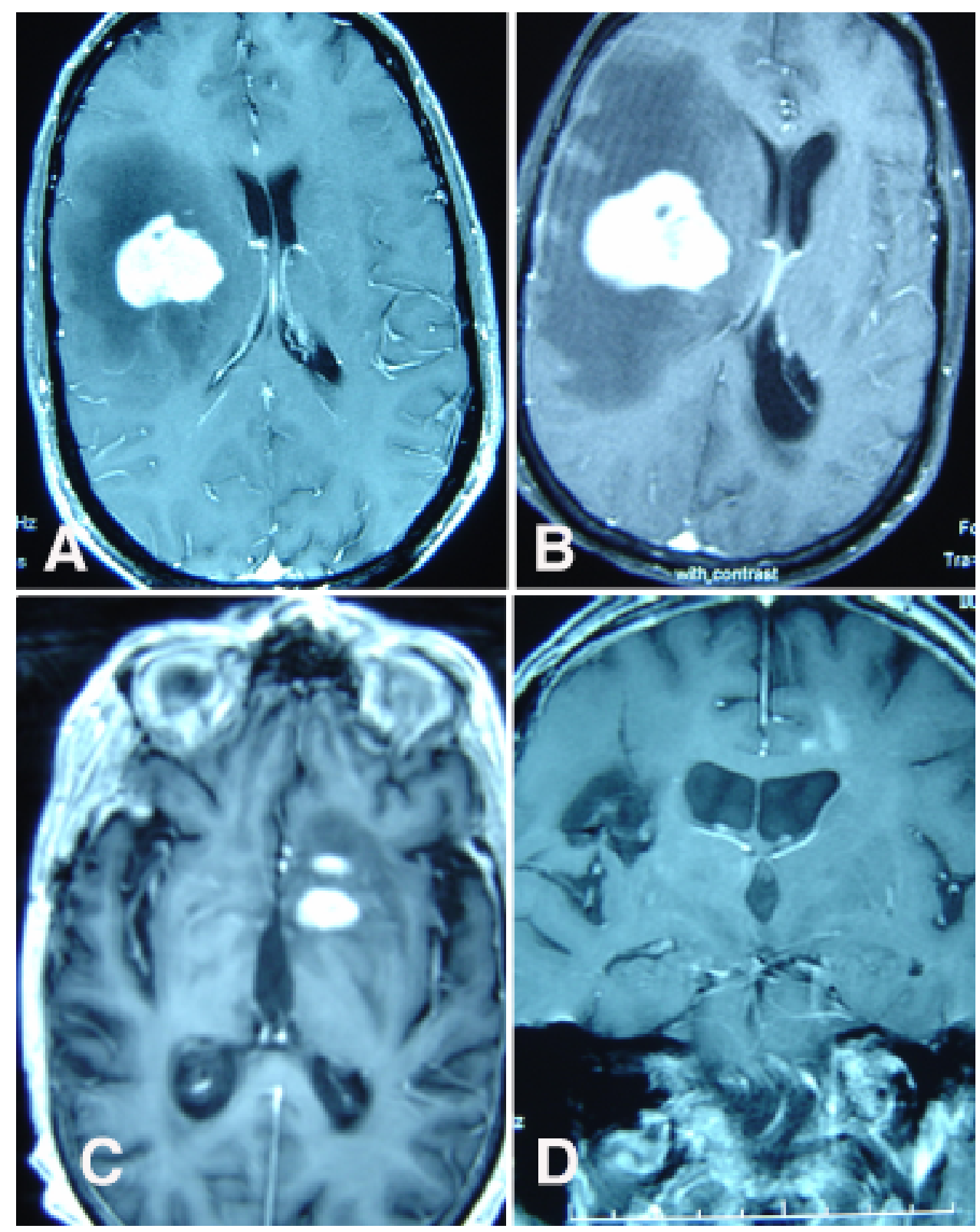

FIG. 1. Case 1. Contrast-enhanced MR images. A: Axial image showing a large enhancing mass in the right hemisphere. Surrounding edema and mild midline shift are also evident. B: Axial image obtained after rapid deterioration of the patient's neurological condition revealing an increase in the tumor size and worsening of the associated edema and midline shift. C and D: Axial (C) and coronal (D) images obtained 9 months after craniotomy showing multiple new lesions in the left hemisphere. The resection cavity is visible and contains no enhancing lesions.

mentia presented to an outside facility with a 3-week history of increased memory loss, headache, nausea, and vomiting. She was fully oriented and able to carry on conversation normally, but scored 25/30 on a Mini-Mental State examination. Cranial nerve examination revealed no abnormality, and the results of examination of motor and sensory functions were otherwise also normal. The workup included CT of the head with contrast (Fig. 2A), MR imaging of the brain (Fig. 2B), and lumbar puncture. The patient was transferred to our hospital with a diagnosis of ventriculitis.

Review of the patient's MR imaging by neuroradiolo- gists at our hospital yielded a high suspicion for lymphoma. Additional studies included MR spectroscopy, which showed decreased $N$-acetylaspartate and increased choline peaks. The results of a whole-body PET study were negative for extracranial disease. The results of an ophthalmologic examination, CT scans of the chest, abdomen, and pelvis, and an HIV test were also negative. Cytological analysis of CSF obtained via lumbar puncture was also suggestive of lymphoma, but tissue diagnosis was recommended to further classify the tumor. A stereotactic brain biopsy of the periventricular lesion at the trigone was positive for B-cell lymphoma. Prior to transfer to a cancer 


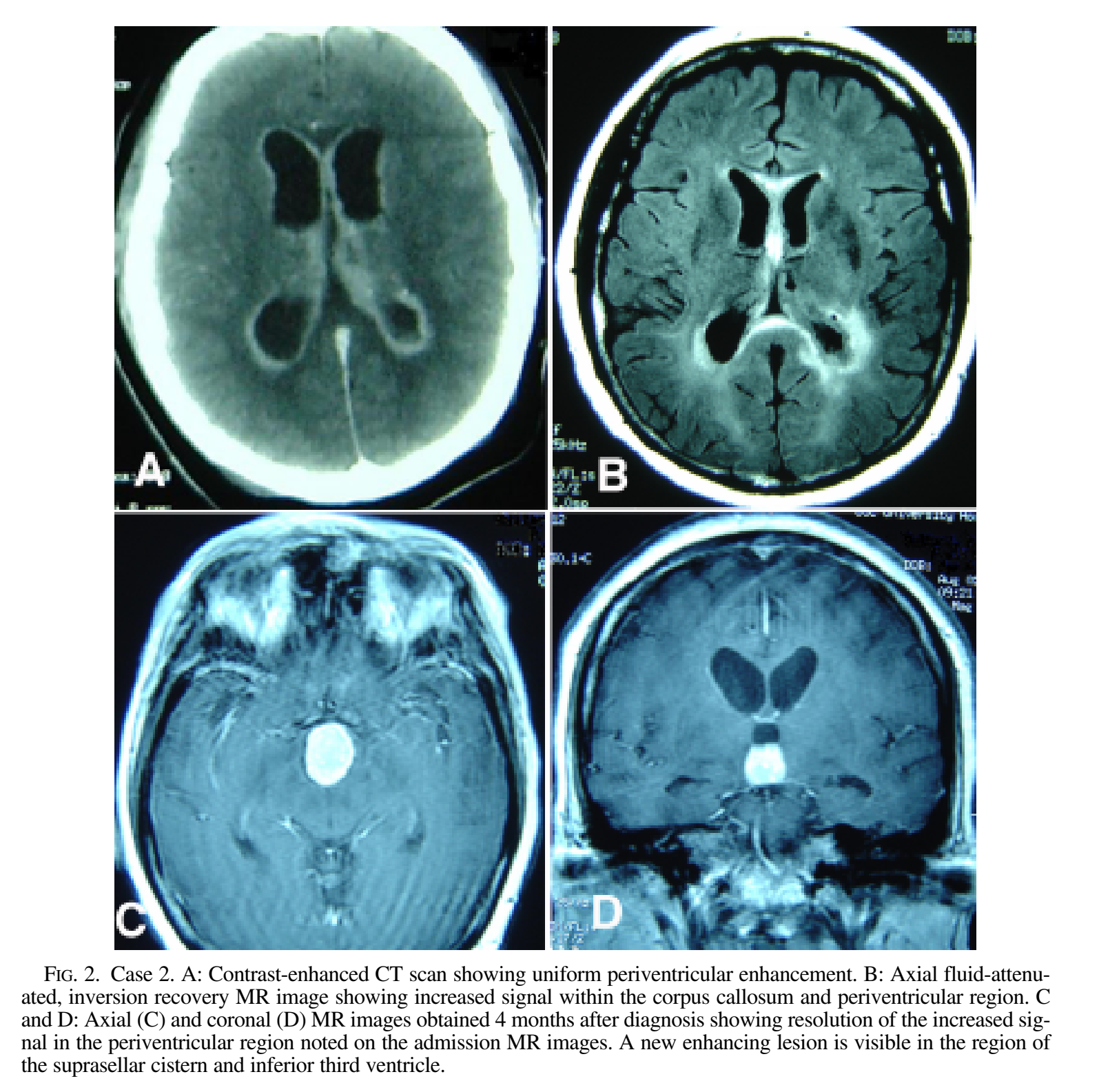

hospital, the patient underwent placement of an Ommaya reservoir.

Follow-up imaging 4 months after diagnosis showed resolution of the majority of the periventricular signal changes (Fig. 2C and D). However, a mass lesion in the suprasellar cistern and third ventricle was noted as a new finding.

\section{Discussion}

Stereotactic biopsy is considered by most authors to be the only surgical intervention indicated for patients with PCNSL. Resection is usually not considered due to the multicentric, infiltrative nature of the tumor and the usual dramatic response to chemotherapy. Gross-total resection or debulking of PCNSL is widely believed to offer no additional clinical or survival benefit over biopsy alone..$^{1,3}$ In an article published in 1986, Murray and colleagues ${ }^{19}$ reported a series of 11 cases of PCNSL and reviewed the previously reported cases - 86 case reports, which included 693 cases of PCNSL (Table 1). In 398 of the cases, the patients were treated with craniotomies, and 85 of these patients had no postoperative adjuvant therapy. The median reported survival time among these 85 patients was 1 month. Only one patient survived beyond 3 years. The authors concluded that extent of resection had no effect on median survival. The only favorable therapeutic variable was a radiation treatment dose greater than $50 \mathrm{~Gy}$.

Authors of more recent studies continue to conclude that biopsy is the sole surgical intervention indicated in the treatment of PCNSL. Tomlinson and coauthors ${ }^{25}$ report their experience with 89 cases of PCNSL. Craniotomy was performed in 42 patients, and the remainder underwent biopsy. Survival was not significantly different between the groups, and the authors concluded that extent of resection was not associated with survival. However, interpretation of this data is difficult because no selection criteria for the craniotomy patients were provided. On the 
TABLE 1

Craniotomy for PCNSL*

\begin{tabular}{|c|c|c|c|c|c|c|c|c|}
\hline \multirow[b]{2}{*}{ Author, Yr } & \multirow[b]{2}{*}{ No. of Patients } & \multirow[b]{2}{*}{ Study Type } & \multirow[b]{2}{*}{ Craniotomy } & \multicolumn{3}{|c|}{ Extent of Resection } & \multicolumn{2}{|c|}{ Postop Survival } \\
\hline & & & & Subtotal & Gross Total & Unknown & Craniotomy & Biopsy \\
\hline $\begin{array}{l}\text { Murray } \\
\text { et al., } 1986\end{array}$ & 693 & lit rev & 398 & - & - & - & $\begin{array}{c}\text { median }=1 \text { mo for } \\
85 \text { pts w/out adj ther }\end{array}$ & NA \\
\hline $\begin{array}{l}\text { Murray } \\
\text { et al.,1986 }\end{array}$ & 11 & retr chart rev & 7 & 3 & 4 & - & $\begin{array}{l}53 \text { mos for pts w/ } \\
\text { gross-total resection; } \\
13 \text { mos for pts w/ } \\
\text { subtotal resection }\end{array}$ & $15 \operatorname{mos}$ \\
\hline $\begin{array}{l}\text { Davies } \\
\text { et al.,1994 }\end{array}$ & 1 & retr chart rev & 1 & 0 & 1 & 0 & $240 \mathrm{mos}$ & NA \\
\hline $\begin{array}{l}\text { Tomlinson } \\
\text { et al., } 1995\end{array}$ & 89 & retr chart rev & 42 & - & - & - & $16.8 \mathrm{mos}$ & $24.5 \operatorname{mos}$ \\
\hline $\begin{array}{l}\text { Sonstein } \\
\text { et al., } 1998\end{array}$ & 1 & retr chart rev & 1 & 0 & 1 & 0 & $60 \mathrm{mos}$ & NA \\
\hline $\begin{array}{l}\text { Bataille } \\
\text { et al., } 2000\end{array}$ & 248 & retr chart rev & 116 & 45 & 66 & 5 & $\begin{array}{l}56.6 \% \text { of pts } \\
\text { alive at } 12 \mathrm{mos}\end{array}$ & $\begin{array}{l}48.6 \% \text { of pts } \\
\text { alive at } 12 \mathrm{mos}\end{array}$ \\
\hline $\begin{array}{l}\text { Bellinzona } \\
\text { et al., } 2005\end{array}$ & 32 & retr chart rev & 25 & 10 & 15 & 0 & NA & NA \\
\hline
\end{tabular}

* adj = adjuvant; lit = literature; retr = retrospective; pts = patients; rev = review; NA = not applicable; ther = therapy; $-=$ data not provided.

basis of a retrospective analysis of PCNSL cases, Bataille and colleagues ${ }^{3}$ have contended that open craniotomy with partial tumor resection is contraindicated. Of the 248 patients in the study, $66(27 \%)$ underwent complete resection of their tumors. The 1-year survival rate in this group was $56.6 \%$, compared with $48.6 \%$ in patients who had stereotactic biopsy alone, and $31.8 \%$ in those who underwent partial resection. Among the three treatment variables, only partial resection achieved a statistically significant, albeit negative, impact on survival. The authors do not discuss the group of patients who underwent complete resection of their tumors and whose 1-year survival rate, although not statistically significant, is suggestive of possible therapeutic benefit.

Factors that are important to evaluate when considering craniotomy for resection of a suspected PCNSL lesion include location, size, and number of lesions present. A recent report ${ }^{5}$ supports the role of craniotomy in a select subset of patients who present with large, solitary, spaceoccupying lesions in the setting of neurological deterioration. The authors describe 33 cases of PCNSL in 32 patients. In this group, 23 patients presented with clinical signs of high intracranial pressure. The mean tumor size at diagnosis was $4.1 \mathrm{~cm}$. Of the 25 patients who underwent craniotomy, 15 had gross-total resection and 10 had partial resection. The remaining eight patients underwent stereotactic biopsy. No specific details regarding patient selection for the surgical options were provided. The authors concluded that radiation therapy and chemotherapy improved survival and that, although surgery provided no long-lasting benefit, a subset of patients may benefit from craniotomy.

\section{Recommendations}

All patients who present with newly diagnosed mass lesions in the brain should be categorized on the basis of clinical presentation, age, and radiographic findings. Steroids should be withheld in the group in whom PCNSL is suspected. This group should then be subdivided according to severity of symptoms. Patients with possible pending herniation and a surgically accessible mass lesion may be considered for urgent craniotomy for debulking of the tumor and tissue diagnosis. Patients without signs of herniation may undergo further preoperative evaluation, including HIV testing, lumbar puncture, ophthalmologic evaluation, and bone marrow biopsy. Patients who are HIV positive should undergo PET scanning and toxoplasmosis titre to differentiate toxoplasmosis from lymphoma. Ultimately, the majority of patients will require stereotactic brain biopsy for tissue diagnosis. If PCNSL is part of the differential, glucocorticoids should be withheld prior to tissue diagnosis, ideally for several days prior to the biopsy procedure. If steroids have been administered prior to the stereotactic biopsy, they should be withheld until the presence or progression of tumor can be verified radiographically or on a PET scan. At this point, tissue can be more reliably obtained via stereotactic brain biopsy.

\section{Summary}

Patients with PCNSL most commonly present with neurological signs and symptoms related to the location of their lesions. The suspicion of lymphoma can be verified by characteristic CT and MRI findings or a history of immunosuppression. If possible, corticosteroids should be avoided prior to obtaining tissue for histological diagnosis. If possible, surgical intervention is limited to a diagnostic stereotactic brain biopsy to obtain tumor specimens for pathological analysis. Chemotherapy, corticosteroids, and whole-brain radiotherapy remain the mainstays of treatment for these tumors. Although the tumors usually show complete remission, relapse typically occurs within 1 year and patients then require salvage therapy. The median survival time after diagnosis is approximately 1 to 4 years. Craniotomy for tumor resection may be indicated in cases in which rapid neurological deterioration or impending herniation is observed. Patients with clinical or radio- 
graphic evidence of large tumor burden who have solitary lesions amenable to resection may represent a subset of patients with PCNSL who merit consideration for open craniotomy to remove their tumors.

\section{References}

1. Abrey LE, Batchelor TT, Ferreri AJM, Gospodarowicz M, Pulczynski J, Zucca E, et al: Report of an international workshop to standardize baseline evaluation and response criteria for primary central nervous system lymphoma. J Clin Oncol 23: 5034-5043, 2005

2. Abrey LE, DeAngelis LM, Yahalom J: Long-term survival in primary CNS lymphoma. J Clin Oncol 16:859-863, 1998

3. Bataille B, Delwail V, Menet E, Vandermarq P, Ingrand P, Wager $M$, et al: Primary intracerebral malignant lymphoma: report of 248 cases. J Neurosurg 92:261-266, 2000

4. Batchelor T, Loeffler JS: Primary CNS lymphoma. J Clin Oncol 24:1281-1288, 2006

5. Bellinzona M, Roser F, Ostertag H, Gaab RM, Saini M: Surgical removal of primary central nervous system lymphomas (PCNSL) presenting as space occupying lesions: a series of 33 cases. Eur J Surg Oncol 31:100-105, 2005

6. Cote TR, Manns A, Hardy CR, Yellin FJ, Hartge P: AIDS/ Cancer Study Group: Epidemiology of brain lymphoma among people with or without acquired immunodeficiency syndrome. J Natl Cancer Inst 88:675-679, 1996

7. Davies KG, Cole GC, Weeks RD: Twenty-year survival following excision of primary CNS lymphoma without radiation therapy: case report. Br J Neurosurg 8:487-491, 1994

8. DeAngelis LM: Primary brain tumors in the acquired immunodeficiency syndrome. Curr Opin Neurol 8:419-423, 1995

9. Ferreri AJM, Reni M, Zoldan MC, Terreni MR, Villa E: Importance of complete staging in non-Hodgkin's lymphoma presenting as a cerebral mass lesion. Cancer 77:827-833, 1996

10. Freeman C, Berg JW, Cutler SJ: Occurrence and prognosis of extranodal lymphomas. Cancer 29:252-260, 1972

11. Geppert M, Ostertag CB, Seitz G, Kiessling M: Glucocorticoid therapy obscures the diagnosis of cerebral lymphoma. Acta Neuropathol 80:629-634, 1990

12. Hao D, DiFrancesco LM, Brasher PMA, deMetz C, Fulton DS, DeAngelis LM, et al: Is primary CNS lymphoma really becoming more common? A population-based study of incidence, clinicopathological features and outcomes in Alberta from 1975 to 1996. Ann Oncol 10:65-70, 1999

13. Herrlinger U, Schabet M, Bitzer M, Petersen D, Krauseneck P: Primary central nervous system lymphoma: from clinical pre- sentation to diagnosis. J Neurooncol 4:219-226, 1999

14. Herrlinger U, Schabet M, Clemens M, Kortmann RD, Peterson $\mathrm{D}$, Will BE, et al: Clinical presentation and therapeutic outcome in 26 patients with primary CNS lymphoma. Acta Neurol Scand 97:257-264, 1998

15. Hochberg FH, Loeffler JS, Prados M: The therapy of primary brain lymphoma. J Neurooncol 10:191-201, 1991

16. Hochberg FH, Miller DC: Primary central nervous system lymphoma. J Neurosurg 68:835-853, 1988

17. Kadan-Lottick NS, Sluzacek MC, Gurney JG: Decreasing incidence rates of primary central nervous system lymphoma. Cancer 95:193-202, 2002

18. Küker W, Nägele T, Korfel A, Heckl S, Thiel E. Bamberg M, et al: Primary central nervous system lymphomas (PCNSL): MRI features at presentation in 100 patients. J Neurooncol 72: 169-177, 2005

19. Murray K, Kun L, Cox J: Primary malignant lymphoma of the central nervous system: results of treatment of 11 cases and review of the literature. J Neurosurg 65:600-607, 1986

20. Pierce MA, Johnson MD, Maciunas RJ, Murray MJ, Allen GS, Harbison MA, et al: Evaluating contrast-enhancing brain lesions in patients with AIDS by using positron emission tomography. Ann Intern Med 123:594-598, 1995

21. Roelcke U, Leenders KL: Positron emission tomography in patients with primary CNS lymphomas. J Neurooncol 43: 231-236, 1999

22. Schabet M: Current developments in primary CNS lymphoma. J Neurooncol 43:197-198, 1999

23. Schabet M: Epidemiology of primary CNS lymphoma. J Neurooncol 43:199-201, 1999

24. Sonstein W, Tabaddor K, Llena JF: Solitary primary CNS lymphoma: long term survival following total resection. Med Oncol 15:61-65, 1998

25. Tomlinson FH, Kurtin PJ, Suman VJ, Scheithauer BW, O'Fallon JR, Kelly PJ, et al: Primary intracerebral malignant lymphoma: a clinicopathological study of 89 patients. J Neurosurg 82:558-566, 1995

26. Weller M: Glucocorticoid treatment of primary CNS lymphoma. J Neurooncol 43:237-239, 1999

This work was supported by a grant from the Marion and Harry Kieper Neurosurgical Research Fund.

Manuscript received September 5, 2006.

Accepted in final form October 4, 2006.

Address reprint requests to: Thomas C. Chen, M.D, Ph.D., 1200 N State Street \#5046, Los Angeles, CA 90033. email: tcchen @usc.edu. 